\title{
Virtual Topology Design in OBS Networks
}

\author{
Mirosław Klinkowski*, Pedro Pedroso ${ }^{\dagger}$, Michał Pióro ${ }^{\ddagger, \pm}$, Davide Careglio ${ }^{\dagger}$, Josep Solé-Pareta ${ }^{\dagger}$ \\ *Z-14, National Institute of Telecommunications, 51-501 Wrocław,Poland, mklinkow@itl.waw.pl \\ $\dagger$ CCABA, Universitat Politècnica de Catalunya, 08034 Barcelona, Spain \\ $\ddagger$ Inst. of Telecomm., Warsaw University of Technology, 00-665 Warsaw, Poland \\ ${ }^{ \pm}$Dep. of Comm. Systems, Lund University, P.O. Box 118, SE-22100 Lund, Sweden
}

\begin{abstract}
The problem of burst losses in OBS networks has an impact on the service quality perceived by end users. In order to guarantee certain level of Service Quality (QoS) in terms of burst losses, wavelength resources have to be dimensioned properly. In this paper, we address the problem of the Virtual Topology (VT) design that concerns the establishment of explicit routing paths and the allocation of wavelengths in network links to support connections with QoS guarantees in the OBS network. We consider the GMPLS control plane as an overlying technology which facilitates the establishment of VT on top of physical network topology.
\end{abstract}

Keywords: GMPLS, Network Design, Optical Burst Switching, QoS Routing.

\section{INTRODUCTION}

The Optical Burst Switching (OBS) paradigm has attracted considerable interest as an optical networking architecture for efficient support of IP packet traffic and flexible access to the immense transmission capacity available with optical fibres and the Wavelength Division Multiplexing (WDM) technology. OBS achieves subwavelength granularity by assembling multiple IP packets into bursts and allocating a wavelength for each burst during the time required for its transmission.

In general, OBS is a buffer-less technology and OBS networks belong to the class of loss networks [1]. Indeed the bursts may contend for link resources at core switching nodes and the contention when unresolved leads to burst losses. The problem of burst contention is of fundamental importance in OBS networks. A method to alleviate the problem is to make use of wavelength converters which allow to access a wavelength from a pool of wavelengths. By these means, a contending burst is accepted as long as there are some wavelength resources available during the burst reservation period.

The problem of burst losses has an impact on the service quality perceived by end users. In order to guarantee certain level of Quality (or Grade) of Service (QoS/GoS), in terms of burst losses, wavelength resources in network links have to be dimensioned properly. The key aspect is to determine and allocate a subset of wavelengths, from the entire set of wavelengths available in the link, that will support given class of service.

In this paper, we address a general problem of optimizing wavelength allocation in an OBS network subject to given (absolute) QoS constraints. More specifically, we are looking for such network routing that for a given set of (long-term) traffic demands and end-to-end (e2e) requirements on the burst loss rate minimizes the overall number of allocated wavelengths (i.e., the wavelength usage) in the network. In the reminder of this paper, we will call such a joint routing and wavelength allocation problem the virtual topology (VT) design problem.

We rely on the Generalized Multi-Protocol Label Switching (GMPLS) control plane as an overlying technology which will have all means to establish label switching paths (LSPs), so that to route bursts through the network, and to allocate wavelength resources in network links [2]. To treat the problem of absolute QoS guarantees analytically, we employ the non-reduced load approximation [3] of common OBS network loss model [1]. Modelling assumptions are then represented as a set of constraints in a Mixed Integer Liner Programming (MILP) formulation. Also, as an alternative to the MILP approach, we propose a local-search heuristic algorithm that, as the numerical results show, can provide good sub-optimal solution to the problem.

The reminder of this paper is organized as follows. In Section 2 we present our assumptions regarding the GMPLS/OBS network scenario. In Section 3 we discuss briefly main modelling steps. In Section 4 we formulate the MILP problem. In Section 5 we present the heuristic algorithm. In Section 6 we present numerical and simulation results that allow us to compare the performance of proposed methods and validate our model. Finally, in Section 7 we conclude obtained results.

\section{GMPLS/OBS NETWORK ARCHITECTURE}

In this paper we focus on a GMPLS-enabled OBS network featuring QoS-guaranteed transport services. In such a scenario, we consider the overall network intelligence is moved to the GMPLS control plane while the OBS layer is only responsible for the data burst transmission and local burst contention resolution.

In the considered GMPLS/OBS network architecture, the extended GMPLS control plane lies on top of the actual OBS network controller, setting up, maintaining, reconfiguring and tearing down LSPs according to the client traffic demands and QoS requirements. We assume each LSP corresponds to an explicit routing path to be used to transport data bursts from source to destination. Moreover, the burst loss probability (BLP) can 
be controlled by an adequate dimensioning of network links that belong to those LSPs. In particular, a virtual topology (VT) of LSPs is deployed for each supported QoS level, where each LSP has an access to a sufficiently large amount of wavelengths so that the requested QoS can be provided. Even though wavelengths can be shared among several LSPs, thus fostering the statistical multiplexing of resources in the network, here we assume these can only be shared among LSPs belonging to the same VT.

Such GMPLS/OBS architecture achieves the idea of keeping the switching layer as fast as possible since only simple, local and limited decisions are required (select a wavelength from a given set). Conversely, routing decisions, congestion notifications or protection/restoration actions are moved to the control plane. To this end, current GMPLS protocol stack will have to be properly extended. Nonetheless, such extensions are not the focus of this paper and are left to future studies. The reader may refer to [2] for some preliminary ideas on this topic.

For sake of simplicity, only one class of quality is considered in this work and, accordingly, only a single VT is designed with the methods presented in the following sections. Nonetheless, these methods can be extended to design multiple VTs where each VT supports traffic with different quality guarantees.

\section{NETWORK MODELLING}

We use $\mathcal{G}=(\mathcal{V}, \mathcal{E})$ to denote the graph of an OBS network; the set of nodes is denoted as $\mathcal{V}$, and the set of unidirectional links is denoted as $\mathcal{E}$. Link $e \in \mathcal{E}$ comprises $W_{e}$ wavelengths. Let $W=\max \left\{W_{e}: e \in \mathcal{E}\right\}$.

Let $\mathcal{P}$ denote the set of predefined candidate LSPs, also referred to as paths, between source $s$ and termination $t$ nodes, $s, t \in \mathcal{V}$, and $s \neq t$. Each path $p \in \mathcal{P}$ is identified with a subset $p \subseteq \mathcal{E}$. Adequately, subset $\mathcal{P} e \subseteq \mathcal{P}$ identifies all paths that go through link $e$. Let $\delta=\max \left\{\delta_{p}: p \in \mathcal{P}\right\}$ be the length of the longest path in the network, where $\delta_{p}$ is the length (in hops) of path $p$.

Let $\mathcal{D}$ denote the set of demands with QoS guarantees, where each demand corresponds to a pair of sourcetermination nodes. For each demand $d \in \mathcal{D}, h_{d} \in \mathbb{R}_{+}$denotes the volume of traffic; for convenience, $h_{p}=h_{d}$ for $p \in \mathcal{P}_{d}$.

Let $\mathcal{P}_{d} \subseteq \mathcal{P}$ denote the set of candidate LSPs supporting demand $d ; \mathcal{P}=\bigcup_{d \in D} \mathcal{P}_{d}$. Each subset $\mathcal{P}_{d}$ comprises a (small) number of paths, e.g., $k$ shortest paths, and a burst can follow one of them.

1) Routing: The network applies source-based routing. The selection of path $p$ from set $\mathcal{P}_{d}$ is performed according to a decision variable $x_{p}$ (also referred to as the routing variable). We assume unsplittable routing, in particular, a burst flow is routed over path $p$ iff $x_{p}=1$ and there is only one path $p \in \mathcal{P}_{d}$ such that $x_{p}=1$. Accordingly, traffic $\rho_{p}$ offered to path $p \in \mathcal{P}_{d}$ is calculated as $\rho_{p}=x_{p} h_{d}$.

2) Burst losses: Due to the complexity of the Erlang fixed-point computation in the common OBS network loss model [1], we assume a simplified model based on the non-reduced load calculation [3]. In this model, to estimate traffic load $\rho_{e}$ offered to link $e$, we add up the traffic load $\rho_{p}$ offered to each path $p \in \mathcal{P}$ that crosses this link: $\rho_{e}=\sum_{p \in \mathcal{P}: p \ni e} \rho_{p}=\sum_{p \in \mathcal{P}: p \ni e} x_{p} h_{p}, e \in \mathcal{E}$. The use of such approximation is justified by its accuracy, particularly under low overall burst losses (below $10^{-2}$ ) [3].

Moreover, we take the common assumption in the literature of i.e.d. burst arrivals, i.i.d. burst durations, together with the assumption of the full wavelength conversion capability in network nodes. Accordingly, the Erlang B-loss formula $\mathcal{B}(\rho, w)$ is used to model the probability $B_{e}$ that a burst is lost in link $e$.

3) Burst loss guarantees: We assume each demand belonging to a QoS class has the same e2e burst loss probability $B^{e 2 e}$ requirements. To meet the goal of the e2e QoS for each demand $d \in \mathcal{D}$, we assume that at each link the burst losses are kept below certain level $B^{l i n k}$, i.e., $B_{e} \leq B^{l i n k}, \forall e \in \mathcal{E}$. For the rest of the paper, we consider $B^{l i n k}$ fixed, the same for each link, and determined according to $B^{\text {link }}=1-\left(1-B^{e 2 e}\right)^{1 / \delta}$. This model is a common model frequently used to assure QoS guarantees in loss networks and it is also applicable in OBS networks under unsplittable source routing.

4) Wavelength allocation: We consider each QoS class has a number of wavelengths allocated in network links which are not shared with other QoS classes. Although, in this paper, we focus on a single QoS class, still such an approach allows to extend the model easily to the scenario with multiple QoS classes.

The last modelling step is to define a dimensioning function $F_{e}(\cdot)$ which for given traffic load $\rho_{e}$ determines the minimum number of wavelengths to be allocated in link $e$ so that to satisfy the blocking $B^{\text {link }}$ requirements. Such a function is given by a discrete (discontinuous, step-increasing) link dimensioning function $F_{e}\left(\rho_{e}\right)=$ $\left[\mathcal{B}^{-1}\left(\rho_{e}, B^{\text {link }}\right)\right]$, where $\mathcal{B}^{-1}\left(\rho_{e}, B^{\text {link }}\right)$ is the inverse of the Erlang B Loss formula extended to the real domain $[4]$, and $\lceil\cdot\rceil$ is the ceiling function.

\section{MILP FORMULATION}

It is convenient to define $a_{w}$ as the maximal load supported by $w$ wavelengths given target blocking probability $B^{\text {link }}$, i.e., $a_{w}=\mathcal{B}^{-1}\left(w, B^{\text {link }}\right)$. Although there is no close formula to calculate $\mathcal{B}^{-1}$, still we can use a line search method (see e.g., [5]) to find the root $\rho^{*}$ of function $f(\rho)=B^{\text {link }}-\mathcal{B}(\rho, w)$ so that to approximate the value of $a_{w}$ by $a_{w}=\rho^{*}$ for each $w \leq W$. Also, we introduce a segmentation on load segments: $b_{w}=$ $a_{w}-a_{w-1}, w=1 \ldots W$. 
Finally, we substitute $F_{e}(\cdot)$ with its piecewise linear approximation, $F_{e}\left(\rho_{e}\right)=\min \left\{w: a_{w} \geq \rho_{e}\right\}$, which further allows us to express the dimensioning function by means of a 0-1 integer programming (IP) formulation. This formulation makes use of a set of binary variables $\left\{u_{e}^{w}: e \in \mathcal{E}, w=1 \ldots W_{e}\right\} ; u_{e}^{w}$ is active iff $w$ or more wavelengths are allocated in link $e$.

Consequently, our VT design problem can be formulated as a MILP problem:

$$
\begin{gathered}
\text { minimize } \quad \sum_{e} \sum_{w} u_{e}^{w} \\
\text { subject to } \quad \sum_{p \in \mathcal{P}_{d}} x_{p}=1, \quad \forall d \in \mathcal{D}, \\
\sum_{p \in \mathcal{P}: p \ni e} h_{p} x_{p}-\rho_{e}=0, \quad \forall e \in \mathcal{E}, \\
\rho_{e} \leq a_{W_{e}}, \quad \forall e \in \mathcal{E}, \\
\sum_{w=1 . . W_{e}} u_{e}^{w} b_{w}-\rho_{e} \geq 0, \quad \forall e \in \mathcal{E}, \\
u_{e}^{w}-u_{e}^{w+1} \geq 0, \quad \forall e \in \mathcal{E}, w=1 . . W_{e}-1, \\
\boldsymbol{u} \in\{0,1\}^{|\mathcal{E}| \times W}, \boldsymbol{x} \in\{0,1\}^{|\mathcal{P}|}, \boldsymbol{\rho} \in \mathbb{R}_{+}^{|\mathcal{E}|} .
\end{gathered}
$$

where $\rho_{e}$ is an auxiliary variable representing load in link $e$.

The objective of the optimization problem is to minimize the total number of wavelengths utilized in the network. (1a) are the routing constraints. (1b) are auxiliary constraints of the non-reduced load calculation. (1c) are the link capacity constraints. (1d) and (1e) result from the 0-1 representation of function $F_{e}(\cdot)$. In particular, the number of wavelengths in link $e$ should be such that the maximum traffic load it can support (calculated as the sum of active load segments $b_{w}$ ) is greater or equal to offered traffic load $\rho_{e}$. Besides, (1e) are ordering constraints, i.e., if $w$ wavelengths are utilized so $w-1$ wavelengths are utilized as well. Finally, (1f) are the variable range constraints.

Note that MILP1 is a variant of the well-known discrete cost multicommodity flow (DCMCF) problem which is a difficult problem [6].

\section{HEURISTIC ALGORITHM}

As an alternative to the MILP approach discussed in Section 4, in this Section we propose a local search (LS) heuristic algorithm. Typically for this type of algorithms, the heuristic starts with a feasible solution and it searches for improved solutions in consecutive iterations. At each iteration a number of solutions neighboring to the so far best solution is checked. In the proposed algorithm we assume a neighboring solution is achieved by means of a flip operation which concerns a permutation of active routing paths of selected demands. The heuristic makes use of the method proposed by Kernighan and Lin [7] for generating neighboring solutions. According, at each iteration very long sequences of flips are considered, even while it appears to be making things worse, in the hope that some neighboring solution will allow to escape from the traps of the local optimum. In the following, we discuss the algorithm details.

Similarly as in MILP, the objective of LS is to improve the overall wavelength usage in the network, denoted as $U(\boldsymbol{x})$, where $\boldsymbol{x}=\left[x_{1} \ldots x_{|\mathcal{P}|}\right]$ is the routing vector. Clearly, $U$ is a function of $\boldsymbol{x}$ since $\boldsymbol{x}$ determines unambiguously traffic load offered to network links and, as a consequence, the number of allocated wavelengths, as discussed in Section 3. Accordingly, $U(\boldsymbol{x})=\sum_{e \in \mathcal{E}} F_{e}\left(\rho_{e}(\boldsymbol{x})\right)$. To compute $F_{e}\left(\rho_{e}\right)$, a simple polynomialtime algorithm, which searches for the lowest $w$ such that $a_{w} \geq \rho_{e}(\boldsymbol{x})$, can be applied. Notice that the routing vector which results in link overload will lead to infeasibility. In such case, we assume $U(\boldsymbol{x})=\infty$.

Let the single-flip neighborhood of routing vector $\boldsymbol{x}$ with respect to demand $d$, denoted as $\lceil\boldsymbol{x}\rfloor_{d(q)}$, be such vector $\hat{\boldsymbol{x}}$ that $\hat{x}_{p}=0$ if $x_{p}=1, p \in \mathcal{P}_{d}$, then $\hat{x}_{q}=1$ for some $q \in \mathcal{P}_{d}, q \neq p$, and $\hat{x}_{r}=x_{r}$ for the rest of paths $r \in \mathcal{P}, r \neq p, r \neq q$. Let $\Omega$ be the set of demands that have not been the subject of the flip operation during the algorithm performance; initially $\Omega=\mathcal{D}$.

A feasible solution $\boldsymbol{x}_{0}$ to start with can be found by solving the following ILP problem:

$$
\begin{gathered}
\text { minimize } \quad 0 \\
\text { subject to } \\
\sum_{p \in \mathcal{P}_{d}} x_{p}=1, \quad \forall d \in \mathcal{D}, \\
\sum_{p \in \mathcal{P}: p \ni e} h_{p} x_{p} \leq a_{W_{e}}, \quad \forall e \in \mathcal{E}, \\
\boldsymbol{x} \in\{0,1\}^{|\mathcal{P}|} .
\end{gathered}
$$

Since the objective function is constant, either a feasible routing vector that satisfies both routing (2b) and link capacity (2c) constraints is found or a notification that such solution does not exist is returned by the solver. 


\begin{tabular}{|c|c|c|c|c|c|c|c|c|c|c|c|c|}
\hline \multicolumn{7}{|c|}{ Scenario } & \multicolumn{3}{|c|}{ MILP } & \multicolumn{2}{|c|}{ LS } & \multirow[b]{2}{*}{ Diff $[\%]$} \\
\hline No & Network & Paths & Traffic & $B^{e 2 e}$ & $W$ & $\rho$ & Usage & Gap [\%] & Time [sec] & Usage & Time [sec] & \\
\hline 1 & SIMPLE & 2 & $\mathrm{U}$ & $10^{-2}$ & 8 & 0.1 & 52 & 0 & 0.297 & 52 & $<0.01$ & 0 \\
\hline 2 & & 2 & $\mathrm{U}$ & $10^{-2}$ & 8 & 0.3 & 88 & 0 & 0.047 & 92 & 0.015 & 4.5 \\
\hline 3 & & 2 & $\mathrm{U}$ & $10^{-2}$ & 32 & 0.1 & 105 & 0 & 2.31 & 107 & $<0.01$ & 1.9 \\
\hline 4 & & 2 & $\mathrm{U}$ & $10^{-2}$ & 32 & 0.3 & 201 & 0 & 14.2 & 203 & 0.016 & 1 \\
\hline 5 & & 2 & $\mathrm{U}$ & $10^{-2}$ & 128 & 0.8 & 1230 & 0 & 4.11 & 1230 & 0.015 & 0 \\
\hline 6 & & 2 & $\mathrm{U}$ & $10^{-3}$ & 64 & 0.5 & 544 & 0 & 17.9 & 544 & 0.015 & 0 \\
\hline 7 & & 2 & NU & $10^{-2}$ & 32 & 0.3 & 202 & 0 & 7.7 & 202 & 0.015 & 0 \\
\hline 8 & & 2 & NU & $10^{-3}$ & 64 & 0.5 & 533 & 0 & 7.8 & 533 & 0.016 & 0 \\
\hline 9 & & 4 & $\mathrm{U}$ & $10^{-2}$ & 32 & 0.3 & 212 & 0 & 171 & 212 & 0.016 & 0 \\
\hline 10 & & 4 & $\mathrm{NU}$ & $10^{-3}$ & 64 & 0.5 & 534 & 0 & 9.6 & 534 & 0.031 & 0 \\
\hline 11 & NSFNET & 2 & $\mathrm{U}$ & $10^{-2}$ & 32 & 0.3 & 729 & 4.9 & 3600 & 738 & 3.78 & 1.2 \\
\hline 12 & & 2 & $\mathrm{U}$ & $10^{-2}$ & 128 & 0.7 & 3975 & 0.98 & 3600 & 3964 & 5.87 & -0.28 \\
\hline 13 & & 2 & NU & $10^{-3}$ & 64 & 0.5 & 1890 & 2.27 & 3600 & 1888 & 2.66 & -0.1 \\
\hline 14 & & 4 & $\mathrm{U}$ & $10^{-2}$ & 32 & 0.3 & 732 & 8 & 3600 & 732 & 8.03 & 0 \\
\hline 15 & UBN & 2 & $\mathrm{U}$ & $10^{-2}$ & 32 & 0.3 & 1456 & 1.34 & 3600 & 1496 & 186 & 2.7 \\
\hline 16 & & 2 & $\mathrm{U}$ & $10^{-2}$ & 128 & 0.3 & 4152 & 1.08 & 3600 & 4163 & 153 & 0.26 \\
\hline 17 & & 2 & $\mathrm{NU}$ & $10^{-3}$ & 64 & 0.3 & 2687 & 1.6 & 3600 & 2706 & 227 & 0.7 \\
\hline 18 & & 4 & $\mathrm{U}$ & $10^{-2}$ & 32 & 0.3 & 1417 & 3.3 & 3600 & 1529 & 451 & 7.9 \\
\hline
\end{tabular}

TABLE I

ALGORITHMS PERFORMANCE.

At each iteration, the main routine of the LS algorithm generates $|\mathcal{D}|$ neighboring solutions. Solution $\boldsymbol{x}_{k}$, where $k=1 \ldots|\mathcal{D}|$, is obtained as the best, among all possible $q \in \mathcal{P}_{d}, d \in \Omega$ and with respect to the usage $U$, single-flip neighborhood $\boldsymbol{x}_{k}=\left\lceil\boldsymbol{x}_{k-1}\right\rfloor_{d(q)}$ of the vector $\boldsymbol{x}_{k-1}$ found in the previous iteration. When a neighborhood is found, the demand $d$ that is the subject to the flip operation is excluded from $\Omega$. When $\Omega$ is empty, the algorithm selects, among all $\boldsymbol{x}_{k}$, an (arbitrary) vector $\boldsymbol{x}^{*}$ such that it minimize the usage, i.e. $\boldsymbol{x}^{*} \in\left\{\boldsymbol{x}_{k}: U\left(\boldsymbol{x}_{k}\right) \leq U\left(\boldsymbol{x}_{m}\right), 0 \leq k \leq|\mathcal{D}|, 0 \leq m \leq|\mathcal{D}|\right\}$. If $U\left(\boldsymbol{x}^{*}\right)<U\left(\boldsymbol{x}_{0}\right)$, a new iteration is started with $\boldsymbol{x}_{0}=\boldsymbol{x}^{*}$ and $\Omega=\mathcal{D}$, otherwise, the algorithm terminates.

An upper bound on the computation time of the main routine of LS is given by $O(W|\mathcal{E}||\mathcal{D}||\mathcal{P}|)$, where $W|\mathcal{E}|$ is a bound on the number of iterations at the worst-case improvement (one per iteration) of the cost function, $|\mathcal{D}|$ is the number of generated neighboring solutions, and $|\mathcal{P}|$ is an upper bound on the number of single-flip candidates that are considered in the search for a neighboring solution. Although the complexity of this routine is polynomial in time, still the feasibility problem ILP1 is $\mathcal{N} \mathcal{P}$-complete (see Proposition 4.2 in [6]). Nevertheless, as the results in Section 6 show, LS can perform quickly.

\section{NUMERICAL RESULTS}

In Table I we present the VT design results obtained for an (arbitrary) set of network scenarios and using the MILP approach and the LS heuristic algorithm. The MILP1 and ILP1 optimization problems are solved by means of the IBM ILOG CPLEX v.12.1 solver [8]. The main routine of the LS algorithm is implemented in Java. The evaluation is performed on a Pentium $\mathrm{M} 2 \mathrm{GHz}$ computer. The performance is expressed in terms of the wavelength usage, the optimality gap, and the computation time. The last column presents a relative difference in the wavelength usage achieved by both algorithms. The results are obtained for SIMPLE (6 nodes, 8 links), NSFNET (15 nodes, 23 links), and UBN (24 nodes, 43 links) mesh network topologies. Considered network scenarios differ in the number of (given per demand) candidate paths, the traffic demand distribution ( $\mathrm{U}$ and NU stand, respectively, for uniform and non-uniform), the e2e BLP requirements $\left(B^{e 2 e}\right)$, the number of available wavelengths $(W)$, and the offered traffic load $(\rho)$. The candidate LSPs are the shortest paths calculated with respect to the number of hops. The capacity of each link is the same and equal to $W$ wavelengths.

For the SIMPLE network, which is a small network, the MILP approach achieves an optimal solution in moderate time ranging from sub-seconds to some tens of seconds. Concurrently, the solution of the LS algorithm in most of the instances is optimal and is achieved in several milliseconds. In both cases, it can be observed that increasing the number of candidate LSPs per demand from 2 to 4 does not necessarily lead to the improvement in the objective value of the wavelength usage (see scenarios 4,9 and 8,10 ). It comes from the fact that in such a small network the length of the longest path may significantly increase and thus the value of $B^{\text {link }}$ decreases (see the modelling details in Section 3). Consequently, more wavelengths are required to accomodate given traffic demands.

For NSFNET and UBN, which are larger networks, we can see that after one hour of solving the MILP problem the optimum is not attained and the optimality gap is between one to a few percents. The increase of the number of candidate paths increases the complexity of the problem (compare the optimality gap in scenarios 11, 14 and 15, 18), however, an improvement in the usage value can be observed in scenario 18 with respect to scenario 15. Eventually, in most cases the LS algorithm is able to find good solution, i.e., comparable with the one given by MILP, in considerably shorter time than when solving the MILP problem. 


\section{CONCLUDING REMARKS}

In this paper, we address the problem of the virtual topology design in OBS networks. The problem concerns the establishment of explicit routing paths and the allocation of wavelengths in network links so that to provide QoS guarantees for the data burst connections. To approach the problem we propose an analytical model which next is used in a MILP formulation. Also, as an alternative method, we present a local-search heuristic algorithm. Although the VT problem is difficult, still proposed methods can provide good solutions, of relatively low optimality gap and in reasonable time, even for larger network scenarios. In order to support changes in network topology and in the traffic demand matrix, in future work we will focus on suitable methods for the problem of VT adaptation under dynamic network scenarios.

\section{ACKNOWLEDGMENT}

This work has been supported by the Polish Ministry of Science and Higher Education under the contract 643/N-COST/2010/0 and by the Spanish Ministry of Science and Innovation through the "COPERNic" project (TEC2009-13252). Pedro Pedroso would like to thank the Portuguese Government Entity, Fundação para a Ciência e a Tecnologia (FCT), for the grant SFRH / BD / 36950 / 2007.

\section{REFERENCES}

[1] Z. Rosberg et al., "Performance Analyses of Optical Burst Switching Networks", IEEE J. Sel. Areas in Commun., vol. 21, no. 7, Sep. 2003, pp. 1187-1197.

[2] P. Pedroso, D. Careglio, R. Casellas, M. Klinkowski, J. Solé-Pareta, “An interoperable GMPLS/OBS Control Plane: RSVP and OSPF extensions proposal", in Proc. PCSN2008, Graz, Austria, July 2008.

[3] M. Klinkowski et al., Graphs and Algorithms in Communication Networks - Studies in Broadband, Optical, Wireless, and Ad Hoc Networks. pp. 165-181, (ed.) A. Koster and X. Muñoz, Springer-Verlag, 2009, ISBN 978-3-642-02249-4.

[4] R. Syski, Introduction to Congestion Theory in Telephone Systems, North-Holland, 1960.

[5] M. Minoux, Mathematical Programming: Theory and Algorithms, John Wiley and Sons, 1986.

[6] M. Pióro and D. Medhi, Routing, Flow, and Capacity Design in Communication and Computer Networks, Morgan Kaufmann, 2004.

[7] B. Kernighan and S. Lin, “An efficient heuristic procedure for partitioning graphs”, The Bell System Technical Journal, vol. 49, no. 2, Feb. 1970, pp. 291-307.

[8] "IBM ILOG CPLEX: High-performance mathematical programming engine.", http://www01.ibm.com/software/integration/optimization/cplex/, 2010. 